Letter: The link between the Reynolds shear stress and the large structures of turbulent Couette-Poiseuille flow

Sergio Gandía-Barberá, Sergio Hoyas, Martin Oberlack, and Stefanie Kraheberger

Citation: Physics of Fluids 30, 041702 (2018); doi: 10.1063/1.5028324

View online: https://doi.org/10.1063/1.5028324

View Table of Contents: http://aip.scitation.org/toc/phf/30/4

Published by the American Institute of Physics

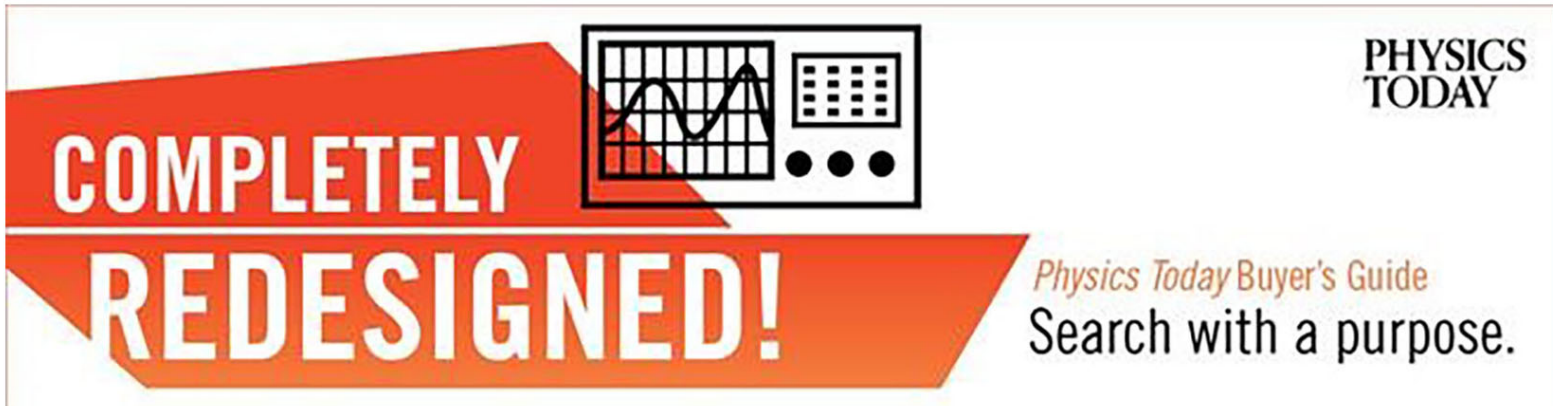




\title{
Letter: The link between the Reynolds shear stress and the large structures of turbulent Couette-Poiseuille flow
}

\author{
Sergio Gandía-Barberá, ${ }^{1}$ Sergio Hoyas, ${ }^{1, a)}$ Martin Oberlack, ${ }^{2}$ and Stefanie Kraheberger ${ }^{2}$ \\ ${ }^{1}$ Instituto Universitario de Matemática Pura y Aplicada, Universitat Politècnica de València, València, Spain \\ ${ }^{2}$ Chair of Fluid Dynamics, TU Darmstadt, Otto-Berndt-Str. 2, 64287 Darmstadt, Germany and Graduate School \\ of Excellence Computational Engineering, TU Darmstadt, Dolivostrasse 15, 64293 Darmstadt, Germany
}

(Received 9 March 2018; accepted 10 April 2018; published online 20 April 2018)

\begin{abstract}
The length and width of the long and wide structures appearing in turbulent Couette flows are studied by means of a new dataset of direct numerical simulation covering a stepped transition from pure Couette flow to pure Poiseuille one, at $R e_{\tau} \approx 130$, based on the stationary wall. The existence of these structures is linked to the averaged Reynolds stress, $\overline{u v}$ : as soon as in any part of the channel $\overline{u v}$ changes its sign, the structures disappear. The length and width of the rolls are found to be, approximately, $50 \mathrm{~h}$ and $2.5 h$, respectively. For this Reynolds number, simulations with a domain shorter than $100 h$ cannot properly describe the behaviour of the longest structures of the flow. Published by AIP Publishing. https://doi.org/10.1063/1.5028324
\end{abstract}

Since the seminal paper of Kim, Moin, and Moser, ${ }^{1}$ turbulent channel flows have been widely studied through direct numerical simulation (DNS). The main control parameter is the friction Reynolds number, $R e_{\tau}=u_{\tau} h / v$, where $u_{\tau}$ is the friction velocity, $h$ is the semi height of the channel, and $v$ is the viscosity. During the last ten years, $R e_{\tau}$ has been consistently increasing in both pressure-driven (Poiseuille, P-flows) ${ }^{2-5}$ and shear (Couette, C-flows) ${ }^{6-8}$ flows. The highest Reynolds number simulated has been very recently published for $R e_{\tau}=8200 .{ }^{5}$ On the other hand, even higher friction Reynolds numbers have been studied experimentally in pipes at $R e_{\tau}=10^{5} .{ }^{9}$ However, the main advantage of DNS is that information on all turbulent scales is available.

Among wall-bounded flows, turbulent $\mathrm{C}$-flows and rotating domains at high rotation numbers ${ }^{10,11}$ have been studied less and at lower Reynolds numbers due to the existence of very large-scale roll-like motions extending along the domain. This fact entails the necessity of long and wide boxes to capture these structures and consequently makes this study more computationally expensive than a turbulent Poiseuille flow at the same Reynolds number. These large coherent structures were found experimentally ${ }^{12-14}$ and numerically. ${ }^{7,8,15,16}$ These structures are defined as coherent regions of either low or high streamwise velocity. They appear as pair, creating counterrotating vortex pairs aligned in the streamwise direction. ${ }^{17}$ There is no clear criterion to identify these rolls, and they are mostly identified through visualization employing some filtering. ${ }^{7,16}$

Additionally, it is believed that they not only have the same qualitative features as near-wall streaks but also cause deviations from the universal wall scaling at sufficiently large Reynolds number, ${ }^{18}$ as secondary flows in ducts do. ${ }^{19}$ Furthermore, these structures are also quite stable. Kraheberger et al. ${ }^{20}$ found that the structures were not completely nullified

a) Author to whom correspondence should be addressed: serhocal@ mot.upv.es by transpiration flows up to $R e_{\tau}=1000$. Lee and $\mathrm{Moser}^{16}$ found out that the streamwise length of these structures depends on the friction Reynolds number, and it is up to $350 \mathrm{~h}$ at $R e_{\tau}=500$. For the flow presented here, at $R e_{\tau}=125$, it is confirmed that there might be structures of this size; however, our main hypothesis is that the largest coherent structures are made up of shorter patterns, whose length is about $50 \mathrm{~h}$. This length is consistent with Lee and Moser ${ }^{16}$ in both span- and stream-wise direction.

The simulation of pure Couette flow at this low Reynolds number is thus comparable to other simulations made by Tsukahara et al. ${ }^{6}$ and Bernardini et $a .^{21}$ Nevertheless, the streamwise size of the boxes must be taken into account while comparing the results. Bernardini ${ }^{21}$ performed DNS at $R e_{\tau}=167$ in a box $(12 \pi h \times 2 h \times 4 \pi h)$. On the contrary, Tsukahara ${ }^{6}$ performed DNS at $R e_{\tau}=126$ in several boxes up to $(64 h \times 2 h \times 6 h)$. Pirozzoli et al. in a very interesting paper ${ }^{18}$ studied the transitions from C- to P-flows at $R e_{\tau}=250$. They related the absence of true large-dynamics scale for the P-flows to the lack of significant transport of turbulent kinetic energy. However, their box had a length of only $12 \pi h$, so the largest structures of C-flows could not be studied in large.

To overcome the problem of the length of the computational boxes, a new set of DNS of the complete transition from pure Couette (i.e., C10P00) to pure Poiseuille flow (i.e., C00P10), see Fig. 1, has been performed for a very long computational box of $L_{x}=128 \pi h, L_{y}=2 h$, and $L_{z}=6 \pi h$,

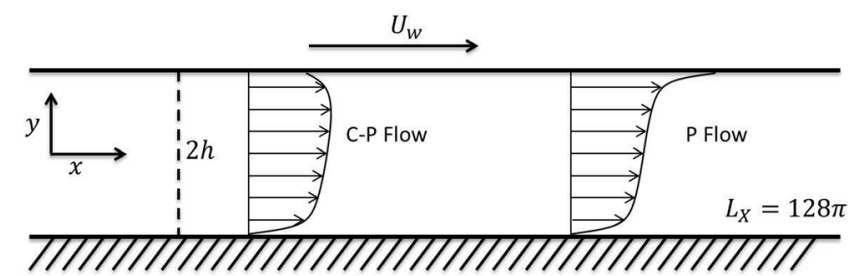

FIG. 1. Schematic view of the different flows with the moving wall velocity $U_{w}$. The computational box is not scaled. 
TABLE I. Parameters of the simulations. Two different Reynolds numbers are given depending on the local $u_{\tau}$ at the stationary (third column) or the moving (fourth) wall. $N_{x}, N_{y}$, and $N_{z}$ are the collocation points in physical space. The last two columns denote the computational time span, while statistics were taken in wash-outs $\left(U_{b} / L_{x}\right)$ and eddy turnovers $\left(u_{\tau} / h\right) . T$ is the computational time spanned by those fields. Line shapes given in the second column are used to identify the cases through all the figures of the present paper.

\begin{tabular}{lcccccccc}
\hline \hline Case & Line & $R e_{\tau}^{s}$ & $R e_{\tau}^{m}$ & $N_{x}$ & $N_{y}$ & $N_{z}$ & $T U_{b} / L_{x}$ & $T u_{\tau} / h$ \\
\hline C10P00 & - - & 132 & 132 & 6144 & 151 & 576 & 8.9 & 188 \\
C08P02 & $\ldots \ldots$ & 133 & 83 & 6144 & 151 & 576 & 10.9 & 235 \\
C06P04 & --- & 135 & 13 & 6144 & 151 & 576 & 11.2 & 243 \\
C04P06 & $-\cdot-$ & 137 & 69 & 6144 & 151 & 576 & 19.4 & 430 \\
C02P08 & - & 142 & 102 & 6144 & 151 & 576 & 16.8 & 384 \\
C00P10 & $-\square-$ & 147 & 147 & 6144 & 151 & 576 & 11.1 & 264 \\
\hline \hline
\end{tabular}

with spanwise and streamwise periodicities. Another set for $L_{x}=16 \pi h$ has also been computed to study the effects of a too short domain. The streamwise, wall-normal, and spanwise coordinates are respectively denoted by $x, y$, and $z$, and the corresponding velocity components are $U, V$, and $W$. Statistically averaged quantities are denoted by an overbar, whereas fluctuating quantities are denoted by lowercase letters, i.e., $U=\bar{U}+u$. The governing equations of the system are transformed into an equation for the wall-normal vorticity $\omega_{y}$ and for the Laplacian of the wall-normal velocity $\phi=\nabla^{2} v$. The spatial discretization uses dealiased Fourier expansions in $x$ and $z$, and seven-point compact finite differences in $y$, with fourth-order consistency and extended spectral-like resolution..$^{22}$ The temporal discretization is a third-order semi-implicit Runge-Kutta scheme. ${ }^{23}$ The code used for this work has been employed in different studies for several different boundary conditions,,$^{2,7,20,24-26}$ so no further validation will be shown here.

For every case, the flow is driven by a constant velocity of the upper wall $(y / h=1)$ and/or a pressure gradient such that the bulk Reynolds number $\operatorname{Re}_{b}=U_{b} h / v$ remains constant at
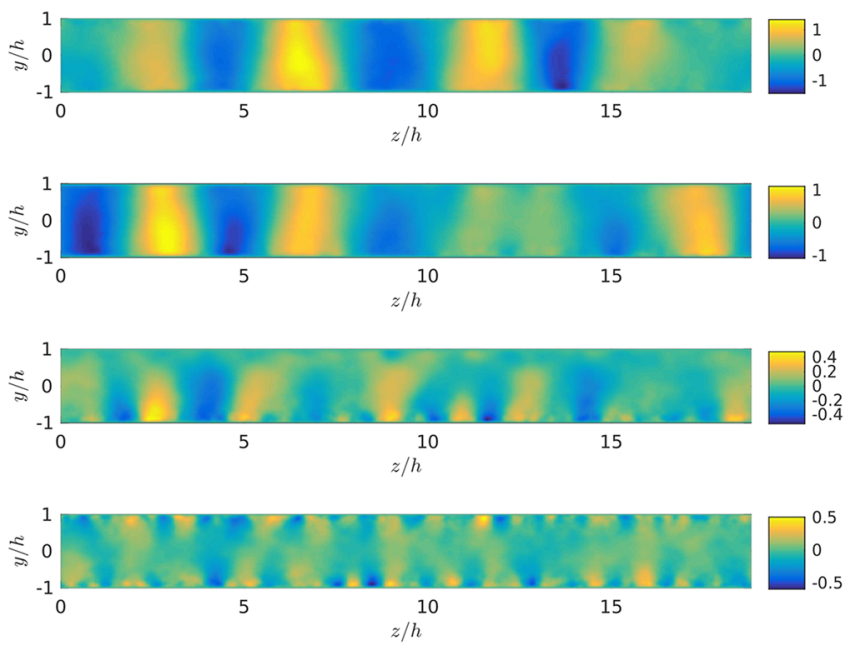

(a)

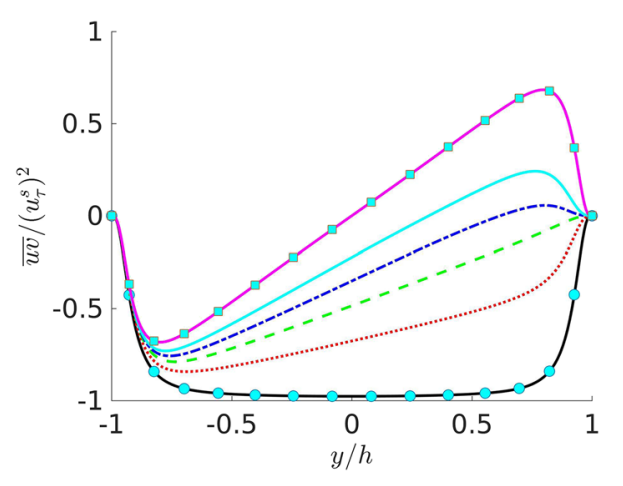

FIG. 2. Lines as in Table I. Reynolds stress scaled by $u_{\tau}^{s}$.

approximately $\operatorname{Re}_{b}=2250$. The lower wall $(y / h=-1)$ is at rest in every case. In every simulation, the flow had to evolve from an initial file, which has been taken from previous different simulations. The code was run until a transition phase was passed, and the flow had adjusted to the new set of parameters. The transition is characterized by, among other variables, the value of the shear stress in the moving wall. Once this parameter reaches a plateau, statistics are collected.

The different simulations are presented in Table I. The wall-normal grid spacing is adjusted to keep the resolution at $\Delta y=1.5 \eta$, i.e., approximately constant in terms of the local isotropic Kolmogorov scale $\eta=\left(v^{3} / \epsilon\right)^{1 / 4}$. In wall units, $\Delta y^{+}$ varies from 0.83 at the wall, up to $\Delta y^{+} \simeq 2.3$ at the centerline. The wall-parallel resolution in physical space for $x$ and $z$ is $\Delta x^{+} \simeq 9.4$ and $\Delta z^{+} \simeq 4.7$, respectively.

Apart from visual inspection, two complementary tools have been used to assert the presence of the rolls. The first one is the two-dimensional spectral energy density, $\Phi=$ $k_{x} k_{z} E\left(k_{x}, k_{z}\right)$, where $k_{x}$ and $k_{z}$ are wave numbers and $E\left(k_{x}, k_{z}\right)$ is the energy spectrum. The second tool is the two point autocorrelation of $U$, in both $\Delta x$ and $\Delta z$.

The different profiles for the Reynolds stress are shown in Fig. 2. They are similar to those of Ref. 18, although the present database has a constant step from C- to P-flow. The
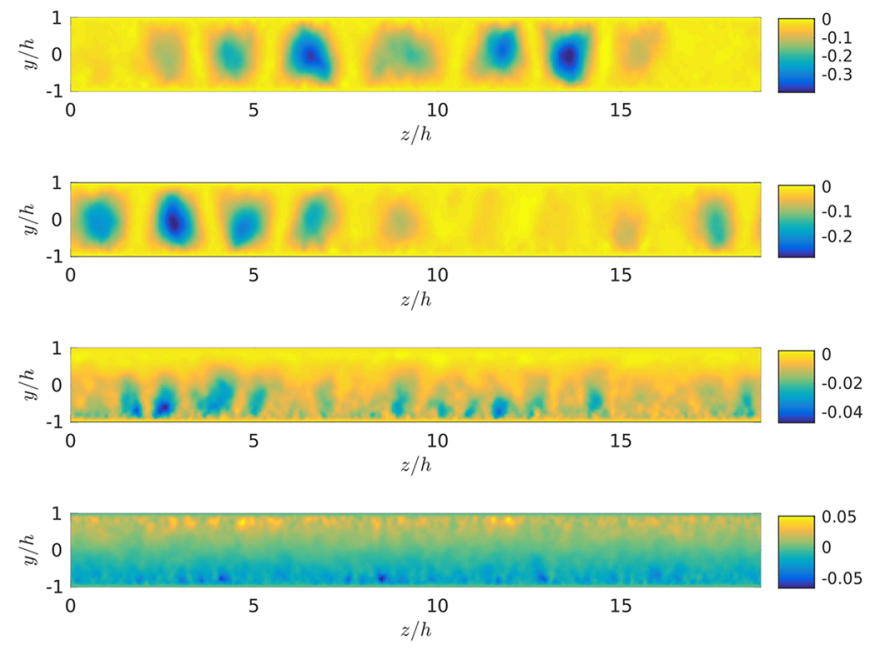

(b)

FIG. 3. Time- and X-averaged images of (a) $u / u_{\tau}$ and (b) $u v / u_{\tau}^{2}$ for the cases, top to bottom, C10P00, C08P02, C04P06, and C00P10. 


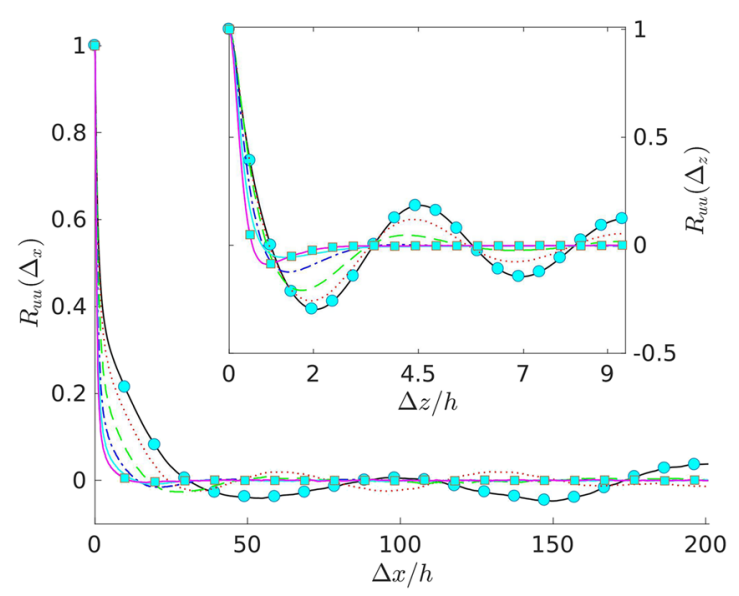

FIG. 4. Lines as in Table I. Autocorrelation of $U$ at $y / h=0.065$ in the streamwise direction (outer box) and spanwise one (inner box).

main hypothesis of this paper is that the existence of the rolls is related to a constant algebraic sign of the averaged Reynolds stress, $\overline{u v}$, through the whole channel.

Figure 3 shows the mean in $x$ of the time-assembled average of $u / u_{\tau}$ [Fig. 3(a)] and $u v / u_{\tau}^{2}$ [Fig. 3(b)] for $L_{x}=16 \pi / h$ database. The rolls leave a clear footprint in both averaged fields. While Fig. 3(a) shows the rolls as pairs of negative and positive $u / u_{\tau}$, Fig. 3(b) relates them to the regions of strong negative values of $u v$. Moreover, this agrees with the conservation of the mean momentum for C-flows, $\tau_{S}=v d \bar{U} / d y-\overline{u v}$, where the changes in $d \bar{U} / d y$ are compensated by $\overline{u v}$. On the other hand, as soon as a pressure gradient appears, $\overline{u v}$ reduces its magnitude. If pressure becomes relevant, as in C06P04 and beyond, the emerging top region of zero $u v$ eliminates the $u v$-rolls and thus the $u$-structures.

At this Reynolds number, this technique cannot be used in the long $L_{x}$ database, as the structures leave no footprint in the ensemble averaged field. Using the autocorrelation of $u$ in $x$, the length of the rolls can be gained from Fig. 4. The extremely long domain employed shows the second minimum of the correlation for the first time, so the length of the longest structures, which is half of the distance between two consecutive minima of the curve, can be estimated as $50 \mathrm{~h}$ approximately. The length of these structures gets shorter when the $\mathrm{C}$ coefficient is reduced, i.e., the $\mathrm{C} 08 \mathrm{P} 02$ case. The different minima cannot be truly appreciated beyond C06P04.

The inner plot of Fig. 4 shows the autocorrelation of $u$ in $z$. The width of the rolls is approximately $2.5 h$. This value is similar to the one in Refs. 7 and 20 but differs from Refs. 6 and 18. These two authors give a smaller value. The main reason for this discrepancy is the width and length of their channel which are not sufficient to simulate a full cycle of the rolls. In Ref. 7, the authors state that the width of the rolls was independent of $R e_{\tau}$, which has been confirmed at $R e_{\tau}=500$ in Ref. 16 and $R e_{\tau}=1000$ in Ref. 20. In both cases, their width coincides with the one found here.

Again, the height of the minima in Fig. 4 goes to zero as the transition to P-flows grows, being inappreciable for the C04P06 case. This is the first case exhibiting negative and positive values of $\overline{u v}$. Thus, it is in agreement with our previously mentioned hypothesis regarding the sign of $\overline{u v}$ and the existence of the rolls.

More information about the width and length of the structures can be obtained from the spectral densities of Figs. 5(a) and 5(b). The thin lines represent $10 \%$ of the maximum energy for every case, while the thick ones represent $75 \%$. The case C10P00 presents a strong maximum in a region centred at $\lambda_{x}=100 h, \lambda_{z}=5 h$, which corresponds to the pairs of rolls described earlier. At this Reynolds number, domains shorter than this length cannot represent adequately the dynamics of the longest structures of C-flow. Moreover, the existence of longer structures cannot be discarded, although with a lower energy footprint. The cases C08P02 and C06P04 also show a strong peak close to the one of $\mathrm{C} 10 \mathrm{P} 00$, but shorter and narrower. At C02P08 and C00P10, the spectra do not exhibit any significant energy in this region, as expected. ${ }^{27}$ The spectral density of $\omega_{y}$ also shows at least a value greater than $10 \%$ of the energy for $\mathrm{C} 10 \mathrm{P} 00$ and $\mathrm{C} 08 \mathrm{P} 02$ at the same region. It is worth noting that C04P06 does not present any energy at this region.

Figure 6(a) shows a filtered field for a short domain of length $16 \pi h$. The structures are long enough to fill the domain. After averaging [Fig. 6(b)], the flow presents a clear strip pattern as a direct consequence of the too short domain and periodic boundary conditions. ${ }^{16}$ When longer boxes are used [Fig. 6(c)], the organization of the flow changes completely as the structures lose streamwise coherence and gain some inclination. The transition between high-speed and low-speed regions can be seen in the red box. In the first half of the box ( $x / h$ from 180 to 240 ), it can be seen two structures: a high-speed one on the top region and a low-speed one on the bottom region. However, in the second half ( $x / h$ from 240 to 280 ), the top region contains a coherent low-speed structure, whereas the bottom one contains the high-speed structure. This vortex dynamics cannot be simulated in boxes with a shorter length.

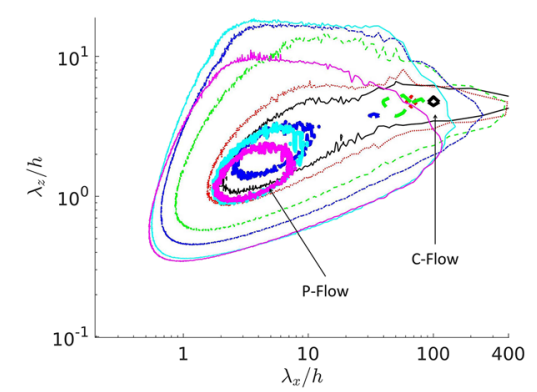

(a)

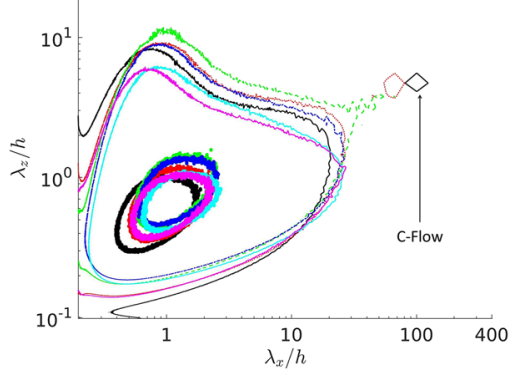

(b)
FIG. 5. Lines as in Table I. Spectral densities at $y / h=$ 0.065 , for (a) $U$ and (b) $y$-component of vorticity, $\omega_{y}$. In both cases, the thin line represents the $10 \%$ of the energy maximum and the thick one the $75 \%$. In every case, $L_{x}=$ $128 \pi h$. 


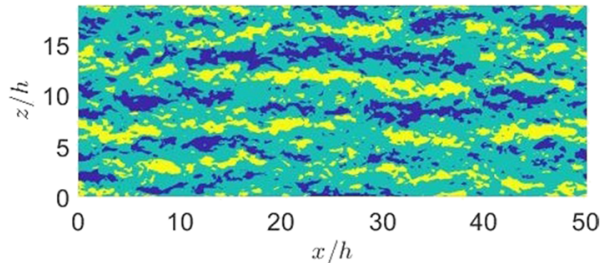

(a)

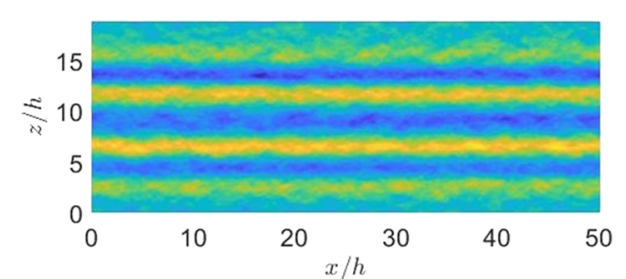

(b)

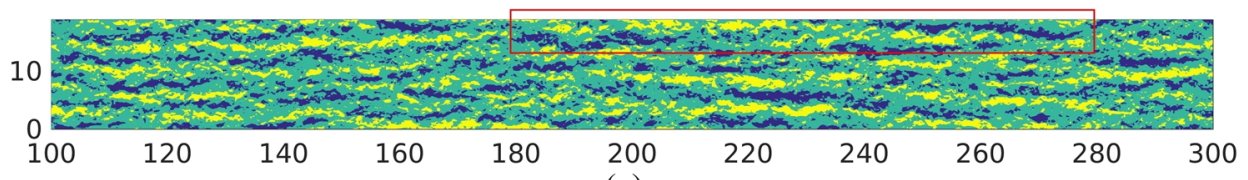

(c)

FIG. 6. (a) Instantaneous filtered $U$ field for pure Couette flow in a $16 \pi h$ box. (b) Ensemble average of $U$ in a $16 \pi h$ box during 10 wash-outs. (c) Instantaneous filtered field of $U$ for a $\mathrm{C} 10 \mathrm{P} 00$ field. Filter defined as follows: values of velocity greater than the intensity $\left(\overline{u^{2}}\right)^{1 / 2}$ : yellow; less than $-\left(\overline{u^{2}}\right)^{1 / 2}$ : blue; and those between these values: green. In every case, the $x-z$ plane is at $y / h=0.065$.
To conclude, at $R e_{\tau}=125$, structures of length $50 \mathrm{~h}$ and width $2.5 h$ have been identified. These low- and high-speed structures develop pairs of counter-rotating vortices, leaving a trace in the $U$ and $\omega_{y}$ spectra. The length and width of the rolls are reduced as the flow becomes more pressure-driven than sheared. As soon as the Reynolds stress does not have a constant sign through the channel, the rolls disappear. This hypothesis is also supported by the fact that in Couette flows with wall transpiration ${ }^{20}$ the Reynolds stress does not change its sign for all the transpiration flows analysed, with the rolls being present in all the flows simulated.

This work was supported by MINECO, under Project No. ENE2015-71333-R. The work of M. Oberlack was supported by the German Research Foundation (DFG) under the Grant No. OB96/39-1. The computations of the new simulations were made possible by a generous grant of computing time from the Supercomputing centre of the Universitat Politècnica de València. We are grateful to Mr. Simón Hoyas for fruitful conversations about the paper.

${ }^{1}$ J. Kim, P. Moin, and R. Moser, "Turbulence statistics in fully developed channels flows at low Reynolds numbers," J. Fluid Mech. 177, 133-166 (1987).

${ }^{2}$ S. Hoyas and J. Jiménez, "Scaling of the velocity fluctuations in turbulent channels up to $R e_{\tau}=2003$," Phys. Fluids 18(1), 011702 (2006).

${ }^{3} \mathrm{M}$. Bernardini, S. Pirozzoli, and P. Orlandi, "Velocity statistics in turbulent channel flow up to $R e_{\tau}=4000$," J. Fluid Mech. 742, 171-191 (2014).

${ }^{4} \mathrm{M}$. Lee and R. Moser, "Direct numerical simulation of turbulent channel flow up to $R e_{\tau} \approx 5200$," J. Fluid Mech. 774, 395-415 (2015).

${ }^{5}$ Y. Yamamoto and Y. Tsuji, "Numerical evidence of logarithmic regions in channel flow at $R e_{\tau}=8000$," Phys. Rev. Fluids 3, 012602(R) (2018).

${ }^{6}$ T. Tsukahara, H. Kawamura, and K. Shingai, "DNS of turbulent Couette flow with emphasis on the large-scale structure in the core region," J. Turbul. 7, N19 (2006).

${ }^{7}$ V. Avsarkisov, S. Hoyas, M. Oberlack, and J. P. García-Galache, "Turbulent plane Couette flow at moderately high Reynolds number," J. Fluid Mech. 751, R1 (2014).

${ }^{8}$ S. Pirozzoli, M. Bernardini, and P. Orlandi, "Turbulence statistics in Couette flow at high Reynolds number," J. Fluid Mech. 758, 327-343 (2014).

${ }^{9}$ M. Hultmark, M. Vallikivi, S. C. C. Bailey, and A. J. Smits, "Turbulent pipe flow at extreme Reynolds numbers," Phys. Rev. Lett. 108, 094501 (2012).

${ }^{10} \mathrm{P}$. Orlandi and M. Fatica, "Direct simulations of turbulent flow in a pipe rotating about its axis,”J. Fluid Mech. 343, 43-72 (1997).
${ }^{11}$ Z. Yang and B.-C. Wang, "Capturing Taylor-Görtler vortices in a streamwise-rotating channel at very high rotation numbers," J. Fluid Mech. 838, 658-689 (2018).

${ }^{12} \mathrm{~N}$. Tillmark, "Experiments on transition and turbulence in plane Couette flow," Ph.D. thesis, KTH, Royal Institute of Technology, 1995.

${ }^{13}$ O. Kitoh, K. Nakabyashi, and F. Nishimura, "Experimental study on mean velocity and turbulence characteristics of plane Couette flow, low-Reynoldsnumber effects and large longitudinal vortical structure," J. Fluid Mech. 539, 199-227 (2005).

${ }^{14} \mathrm{O}$. Kitoh and M. Umeki, "Experimental study on large-scale streak structure in the core region of turbulent plane Couette flow," Phys. Fluids 20(2), 025107 (2008).

${ }^{15} \mathrm{~K}$. Bech, N. Tillmark, P. Alfredsson, and H. Andersson, "An investigation of turbulent plane Couette flow at low Reynolds numbers," J. Fluid Mech. 286, 291-325 (1995).

${ }^{16} \mathrm{M}$. Lee and R. Moser, "Extreme-scale motions in turbulent plane Couette flows," J. Fluid Mech. 842, 128-145 (2018).

${ }^{17}$ S. K. Robinson, "Coherent motions in the turbulent boundary layer," Annu. Rev. Fluid Mech. 23(1), 601-639 (1991).

${ }^{18}$ S. Pirozzoli, M. Bernardini, and P. Orlandi, "Large-scale motions and inner/outer layer interactions in turbulent Couette-Poiseuille flows," J. Fluid Mech. 680, 534-563 (2011).

${ }^{19}$ R. Vinuesa, C. Prus, P. Schlatter, and H. M. Nagib, "Convergence of numerical simulations of turbulent wall-bounded flows and mean cross-flow structure of rectangular ducts," Meccanica 51(12), 3025-3042 (2016).

${ }^{20}$ S. Kraheberger, S. Hoyas, and M. Oberlack, "DNS of a turbulent Couette flow at constant wall transpiration up to $R e_{\tau}=1000$," J. Fluid Mech. 835, 421-443 (2018).

${ }^{21}$ M. Bernardini, S. Pirozzoli, and P. Orlandi, "The effect of large-scale turbulent structures on particle dispersion in wall-bounded flows," Int. J. Multiphase Flow 51, 55-64 (2013).

${ }^{22} \mathrm{~S}$. K. Lele, "Compact finite difference schemes with spectral-like resolution," J. Comput. Phys. 103(1), 16-42 (1992).

${ }^{23}$ P. R. Spalart, R. D. Moser, and M. M. Rogers, "Spectral methods for the Navier-Stokes equations with one infinite and two periodic directions," J. Comput. Phys. 96(2), 297-324 (1991).

${ }^{24} \mathrm{~S}$. Hoyas and J. Jiménez, "Reynolds number effects on the Reynoldsstress budgets in turbulent channels," Phys. Fluids 20(10), 101511 (2008).

${ }^{25}$ V. Avsarkisov, M. Oberlack, and S. Hoyas, "New scaling laws for turbulent Poiseuille flow with wall transpiration," J. Fluid Mech. 746, 99-122 (2014).

${ }^{26}$ F. Lluesma-Rodríguez, S. Hoyas, and M. J. Peréz-Quiles, "Influence of the computational domain on DNS of turbulent heat transfer up to $R e_{\tau}=2000$ for Pr = 0.71, " Int. J. Heat Mass Transfer 122, 983-992 (2018).

${ }^{27}$ A. Lozano-Durán and J. Jiménez, "Effect of the computational domain on direct simulations of turbulent channels up to $R e_{\tau}=4200$," Phys. Fluids 26(1), 011702 (2014). 